\title{
A structural dynamics model for how CPEB3 binding to SUMO2 can regulate translational control in dendritic spines
}

\author{
Xinyu Gu ${ }^{1,2}$, Nicholas P. Schafer ${ }^{1,2}$, Carlos Bueno ${ }^{1}$, Wei Lu ${ }^{1}$, \\ Peter G. Wolynes ${ }^{1,2, *}$ \\ ${ }^{1}$ Center for Theoretical Biological Physics, Rice University, Houston, TX 77005; \\ ${ }^{2}$ Department of Chemistry, Rice University, Houston, TX 77005. \\ E-mail: pw8@rice.edu
}

\section{Abstract}

A prion-like RNA-binding protein, CPEB3, can regulate local translation in dendritic spines. CPEB3 monomers repress translation, whereas CPEB3 aggregates activate translation of its target mRNAs. However, the CPEB3 aggregates, as long-lasting prions, may raise the problem of unregulated translational activation. Here, we propose a computational model of the complex structure between CPEB3 RNA-binding domain (CPEB3-RBD) and small ubiquitin-like modifier protein 2 (SUMO2). Free energy calculations suggest that the allosteric effect of CPEB3-RBD/SUMO2 interaction can amplify the RNA-binding affinity of CPEB3. Combining with previous experimental observations on the SUMOylation mode of CPEB3, this model suggests an equilibrium shift of mRNA from binding to deSUMOylated CPEB3 aggregates to binding to SUMOylated CPEB3 monomers in basal synapses. This work shows how a burst of local translation in synapses can be silenced following a stimulation pulse, and explores the CPEB3/SUMO2 interplay underlying the structural change of synapses and the formation of long-term memories. 
The remodeling of the actin cytoskeleton in dendritic spines serves as the molecular basis for the structural changes of synapses ${ }^{1}$ which are associated with the formation of long-term memories. ${ }^{2}$ A synaptic tag is required to label budding synapses so as to regulate the local translation of actin mRNA and the mRNA of other synaptic proteins over long periods of time. A promising candidate for the synaptic tag is the mammalian cytoplasmic polyadenylation element-binding protein 3 (CPEB3). Mammalian CPEB3 ${ }^{3}$ and its homologs, ApCPEB in Aplysia ${ }^{4}$ and Orb2 in Drosophila, ${ }^{5,6}$ have been shown to regulate the translation of synaptic proteins by binding with the U-rich CPE sequence in the 3' untranslated region (UTR) of target mRNAs. Targets of CPEB3 include the message RNAs for actin ${ }^{7}$ and other protein components essential for long-term synaptic persistence, like GluA1 and GluA2. ${ }^{3}$ The longevity of the synaptic tag is traced to the fact that, CPEB3 can form self-sustaining prion-like aggregates ${ }^{8}$ that resist rapid molecular turnover. ${ }^{9}$ Understanding the system dynamics of the CPEB3-actin regulation network ${ }^{10}$ is essential to see how CPEB3 is able to consolidate synaptic structure and facilitate the formation of long-term memories.

CPEB3 in dendritic spines can be found either in a monomeric state or in an amyloid-like aggregated state. Intriguingly, the monomeric CPEB3 has been found to repress translation, while the aggregated form of CPEB3 activates the translation of its target mRNAs. ${ }^{11-13}$ One idea that has been proposed is that monomeric CPEB3 localizes its target mRNAs through forming gel-like processing bodies ( $\mathrm{P}$ bodies) in which translation is repressed and that CPEB3 aggregation into a prion form simply releases those mRNAs. ${ }^{13}$ A structural study of a CPEB3 homolog, Orb2, has found, however, that Orb2 aggregated in the prion form still binds target mRNAs and interacts with various proteins that might further recruit translation promoting factors. ${ }^{14}$ We have recently suggested that the activation and repression of translation by CPEB3 can be explained by a vectorial channeling effect in which recycling of ribosomes depends on the structure of CPEB3/RNA assemblies. ${ }^{15}$ Vectorial channeling suggests how CPEB3 aggregation may turn on the translation of dormant target mRNAs. The CPEB3 aggregates, which function then as synaptic tags providing "conformational memory", would be stable in synapses. Such stability, by itself would seemingly lead to continuous activation of local translation if aggregates were always found bound with their target mRNAs. This raises the question of whether and how such translational enhancement 
bioRxiv preprint doi: https://doi.org/10.1101/2021.11.24.469918; this version posted November 24, 2021. The copyright holder for this preprint 47 couldwhich was not certified by peer review) is the author/funder. All rights reserveq. No reuse allowed without permission.

One possibility that has been suggested involves the SUMOylation of CPEB3. There is evidence that SUMOylation, a reversible post-translational modification, can regulate CPEB3 function. SUMO proteins, small ubiquitin-like modifier proteins, can be covalently attached to lysine residues of target proteins. After such SUMOylation, the SUMO modification can subsequently be deconjugated (deSUMOylation). Monomeric CPEB3 is found to be SUMOylated and in that form it is soluble in basal synapses. After stimulation, CPEB3 becomes deSUMOylated and aggregates. ${ }^{12}$ SUMOylation of CPEB3 has been found to facilitate its colocalization to $\mathrm{P}$ bodies and is crucial for repressing translation. ${ }^{13}$ DeSUMOylation of CPEB3 exposes the prion-like domain (PRD) and the actin-binding domain (ABD) of CPEB3 and triggers actin-facilitated CPEB3 aggregation as a result. ${ }^{16}$ An interesting fact in thinking about the system biology of SUMO/CPEB3 is that SUMO mRNA itself is one of CPEB3's targets. Therefore, there is a negative feedback loop between CPEB3 aggregation and SUMO synthesis: ${ }^{12}$ The CPEB3 prion activates the translation of SUMO which can then be used for CPEB3 SUMOylation and lead to a return to translational repression. This negative feedback loop could thereby serve as a control on the balance between activation and repression of translation by CPEB3.

In this paper, we put forward a structural dynamics model of the interaction between SUMO2 and the RNA-binding domain (RBD) of SUMOylated CPEB3. We developed a structural model for the SUMO2/CPEB3-RBD complex through computational modeling using the Associative memory, Water-Mediated, Structure and Energy Model (AWSEM), ${ }^{17,18}$ a coarse-grained protein force field which has been optimized using energy landscape theory. ${ }^{19}$ The AWSEM software has proved quite successful in predicting both monomeric protein structures and the structures of protein complexes. ${ }^{17,20}$ The SUMO2 protein simultaneously interacts with two distinct surfaces of the CPEB3 RNA-binding domain. By doing so, it closes the conformation of the RNA binding domain into a structure favorable for RNA binding. Using the AWSEM force field and combining it with the Three Sites Per Nucleotide model 2 (3SPN2), a coarse-grained force field for nucleic acids developed by the de Pablo group,${ }^{21,22}$ we have calculated the free energy profile for RNA dissociation from the SUMO2/CPEB3-RBD complex. These calculations show that the RNA-binding free energy for the SUMO2/CPEB3-RBD complex is $2 \mathrm{kcal} / \mathrm{mol}$ larger than that for isolated RBD in 
ity between the two forms causes a shift in the equilibrium of RNA binding from binding to the deSUMOylated CPEB3 aggregates to binding to SUMOylated CPEB3 monomers when synapses return to a new basal state. In this way, the translational control of CPEB3 becomes switchable in response to input signals: After stimulation, CPEB3 is deSUMOylated, so that CPEB3 aggregates and thereby activates translation of SUMO2 and other synaptic proteins. Monomeric CPEB3 once SUMOylated with newly synthesized SUMO2 proteins shifts the RNA-binding equilibrium, so that target mRNAs are recruited into P bodies by the SUMOylated CPEB3 and thereby are silenced.

\section{Results}

\section{A full-length CPEB3-RBD model details its inter-domain interac- tions}

The CPEB family consists of four isoforms in mammals, CPEB1-4. The composition of the C-terminal RBD in all four isoforms is highly conserved. The RBD includes two RNA recognition motifs (RRMs) and one zinc-finger domain $(\mathrm{ZnF})$ as shown in Fig. 1A. These isoforms can be divided into two distinct subgroups based on the sequence similarity of the RBD: Taking the mouse CPEB family as an example, the RBDs of CPEB2-4 have 96\% pairwise sequence identities, while the CPEB1 RBD has only $42 \%$ pairwise sequence identity with those of CPEB2-4, as shown in Fig. S1A. The RBDs of CPEB3 homologs in other species, like CPEB3 in human, CPEB3 in rat, and Orb2 in Drosophila, have more than $86 \%$ pairwise sequence identity with the RBD of mouse CPEB3, as shown in Fig. S1B. In this paper, we have studied the sequence of mouse CPEB3-RBD since a large fraction of the experimental work has been performed on mouse CPEB3.

As shown in Fig. 1C/D and Fig. 1B, the NMR structures for the $\mathrm{RRMs}^{23}$ and the $\mathrm{ZnF}^{24}$ of some CPEB homologs have been solved. These were used as templates to build the corresponding structures for mouse CPEB3 via Modeller. ${ }^{25}$ The tandem RNA recognition motifs are essential for sequence-specific recognition. They act by forming direct residuebase contacts with target mRNAs, while the zinc-finger domain only contributes to the RNA-binding affinity but not to the specificity of binding. ${ }^{26,27}$ The NMR structures of the 
bioRxiv preprint doi: https://doi.org/10.1101/2021.11.24.469918; this version posted November 24,2021 . The copyright holder for this preprint
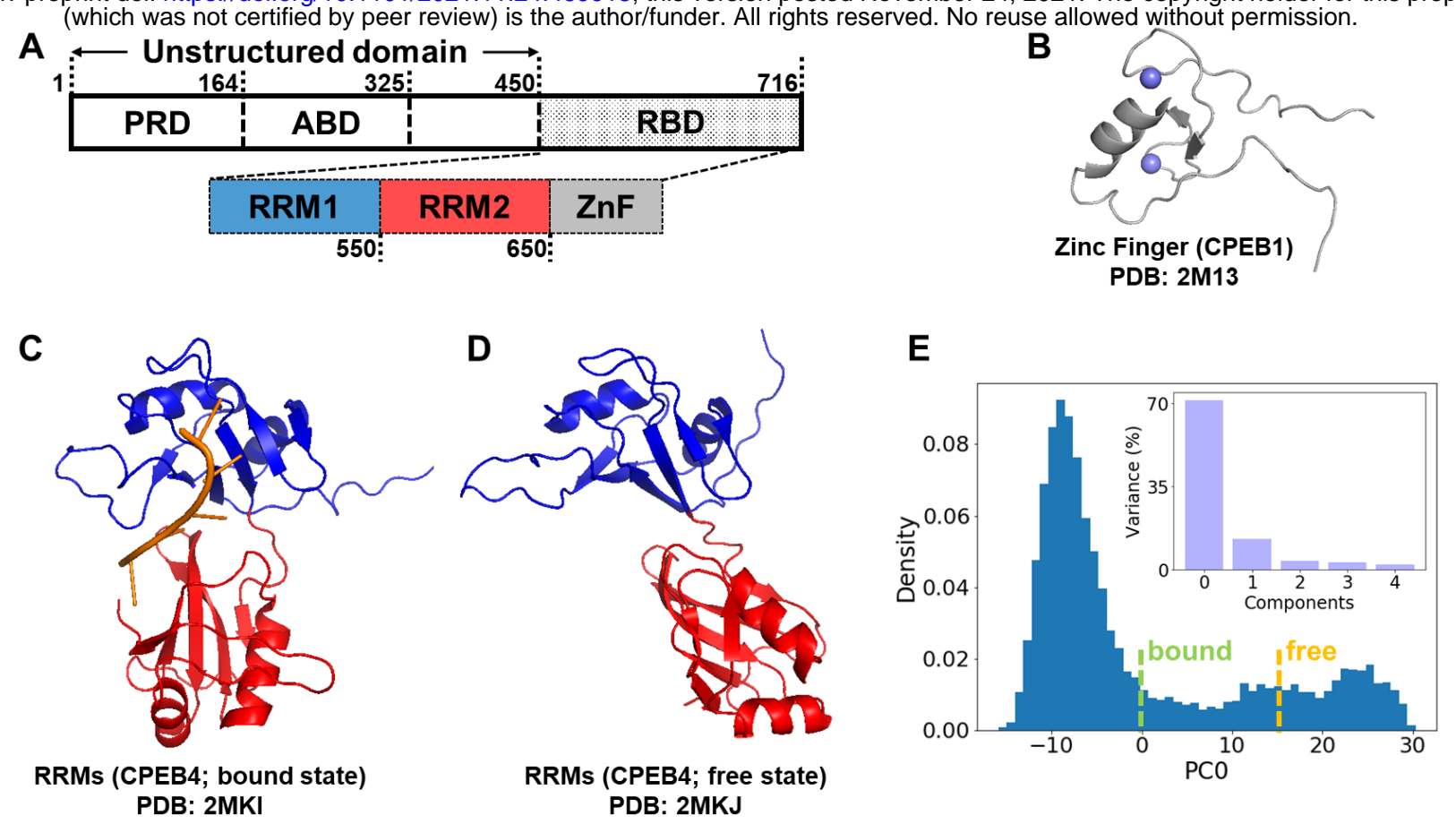

Figure 1: A. A diagram of the CPEB3 subdomains including a prion-like domain (PRD), an actin-binding domain (ABD), and an RNA-binding domain (RBD). The RBD consists of two RNA recognition motifs, RRM1 and RRM2 (colored in blue and red hereinafter), and one Zinc-finger domain (ZnF, colored in grey). B. The NMR structure of CPEB1 ZnF (Zinc ions are shown in purple). C. The NMR structure of CPEB4 RRMs binding with target mRNA (shown in orange). D. The NMR structure of free CPEB4 RRMs. E. The histogram for the distribution of first principal component, PC0, sampled during 20 equilibrium simulation trajectories of CPEB3 RRMs. Embedded: Variance contributed by the first five principal components from the principal component analysis. The PC0 values for the NMR structures of the RNA-bound state and the free state are shown by green and yellow dashed lines, respectively.

RRMs suggest that there is a conformational change from the free state which is in an open conformation to the RNA-bound state which is closed. To study this closure motion, we first ran equilibrium simulations of the free RRMs using the AWSEM force field and conducted a cartesian principal component analysis for all the structures sampled during the equilibrium trajectories. Fig. 1E maps the distribution of all frames onto the most significant principal component, PC0. The distribution displays two distinct modes for this principal component: one mode where $\mathrm{PC} 0$ ranges from -10 to 0 and another mode where $\mathrm{PC} 0$ ranges from 10 to 30. The NMR structure of the bound RRMs has a PC0 value near the first mode while the NMR structure of the free RRMs has a PC0 value near the second mode. We therefore use the $\mathrm{PC} 0$ value as an order parameter to distinguish between the open structures and the closed structures of the RRMs.

To model the full length $\mathrm{RBD}$, we attached the $\mathrm{ZnF}$ domain to the C-terminal of the 
A

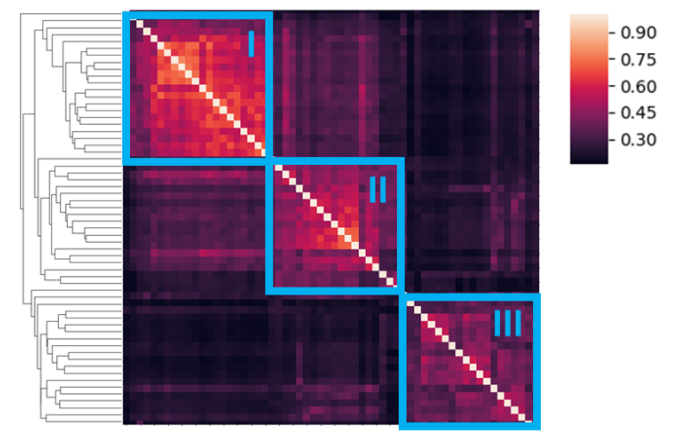

B

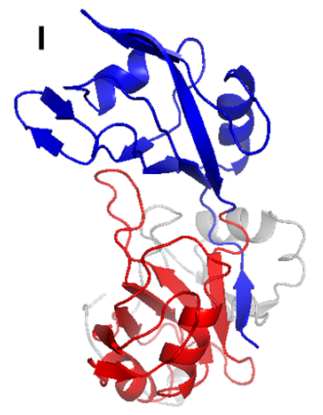

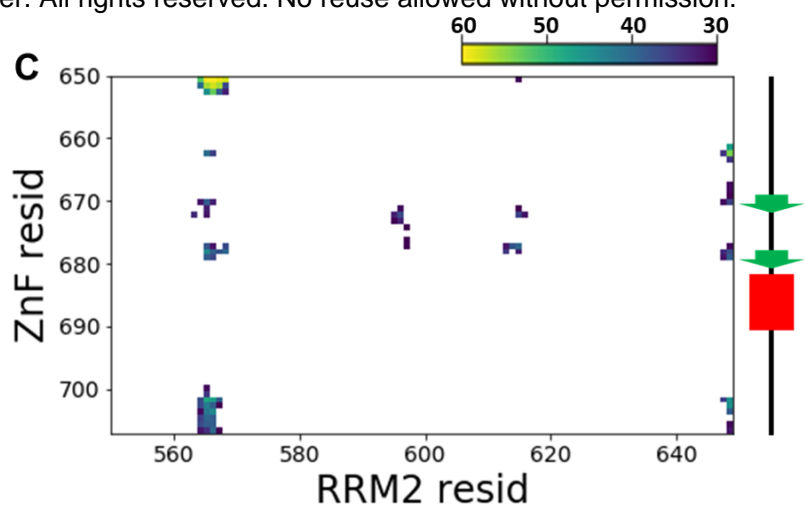

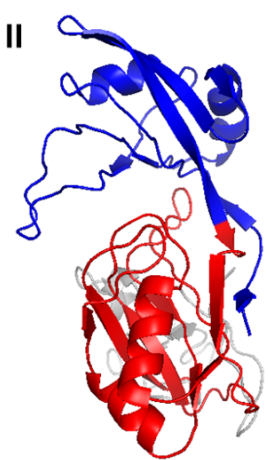

III

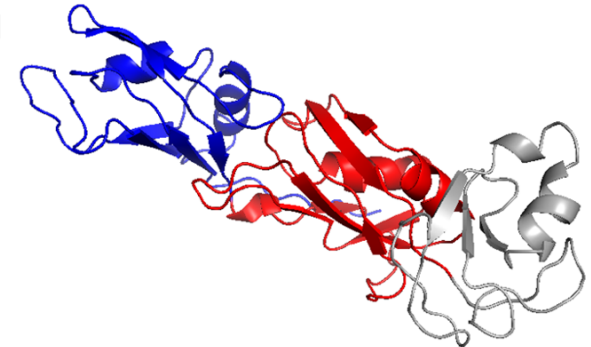

Figure 2: A. The 60 predicted structures of full length RBD that can be divided into 3 clusters using mutual Q (only including inter-domain residue pairs) as the structural similarity metric. B. Representative structures for the 3 clusters in Figure A. C. The frequency contact map for the inter-domain contact between the znic finger domain and the RNA recognition motif 2 (RRM2). Only contacts formed more than 30 times in 60 predictions are shown here. The diagram of the secondary structure of the $\mathrm{ZnF}$ domain is shown on the right side along the y-axis. Beta strands are colored in green and alpha helices are colored in red.

RRMs and ran AWSEM simulations to relax that initial structure. Clustering analysis for the final frames of sixty independent simulations suggests that the full-length RBD can adopt either the open conformation or the closed conformation, as shown in Fig. 2A/B. The probability contact map for ZnF/RRM2 inter-domain contacts (Fig. 2C) shows that the aromatic beta sheet surface (F669, F670, Y679 and Y680) on the ZnF consistently leads to an inter-domain interaction with RRM2 . This interaction between the znic finger domain and the RNA recognition motif 2 was proposed previously based on the NMR data ${ }^{24}$ and we see it emerges also in our model here.

\section{Interaction with SUMO2 guides the RBD to select a conformation that favors RNA binding}

Monomeric CPEB3 is SUMOylated in basal neurons. SUMO2, even after it has become covalently attached to CPEB3, can also non-covalently interact with the SUMO-Interacting 
bioRxiv preprint doi: https://doi.org/10.1101/2021.11.24.469918; this version posted November 24, 2021. The copyright holder for this preprint

teract with the second beta strand, $\beta 2$, of SUMO2 to form an inter-molecular beta sheet. ${ }^{28}$ Bioinformatic searches have identified one potential SIM at an exposed strand in the RNA recognition motif 1 (RRM1) of CPEB3. ${ }^{16}$ The binding energy between this SIM and SUMO2 protein, calculated via the AWSEM Hamiltonian, is relatively large as compared with canonical SIM/SUMO2 complex, as shown in Fig. S2. Thus the interaction between SUMO2 and RRM1 via the SIM is favorable. It is intriguing that the zinc-finger domains from other proteins, such as the ubiquitin ligase HERC2 ${ }^{29}$ and CBP (CREB-binding protein/p300), ${ }^{30}$ have been reported to bind with SUMO proteins, suggesting that the zinc-finger domain contains a SUMO-interacting motif. The RBD of CPEB3, containing one canonical SIM and one zinc-finger domain, therefore may bivalently bind with SUMO2 specifically and stably.

To model the structure of the SUMO2/RBD complex, we first docked SUMO2 to the $\mathrm{ZnF}$ domain in the 60 structures of full-length RBD that were predicted and discussed in the last section while using the NMR structure of the SUMO1/CBP-ZnF complex (PDB ID: 2N1A) as a reference. We then added a weak biasing potential between the RRM1-SIM and the $\beta 2$ strand of SUMO2 to guide the formation of the inter-molecular beta sheet. The resulting structures were then allowed to relax without the use of the biasing potential. Final structures from these relaxation simulations were screened by calculating the inter-molecular structural similarity of RRM1-SIM/SUMO2 to canonical SIM/SUMO2 complex, as shown in Fig. S4. We calculated AWSEM energies and also counts of the number of minimally frustrated contacts ${ }^{31}$ for selected structures as shown in Fig. 3B. In a representative structure of the SUMO2/RBD complex (Fig. 3C), SUMO2 forms two interfaces: one interface formed with the RRM1-SIM and the other formed with the $\mathrm{ZnF}$ domain.

To study the effects of the interaction with SUMO2 on the closure motion of RBD domain, we calculated the free energy profiles for the RBD domain by itself and for the SUMO2/RBD complex (Fig. 4). For the RBD domain alone, there are two free energy basins: one for the open state (basin I in Fig. 4A) and another for the closed state (basin II in Fig. 4A). For the SUMO2/RBD complex in contrast, only the basin of closed state (basin III in Fig. 4B) is found. The bivalent interaction with SUMO2 confines the conformation of CPEB3-RBD domain to the closed state. 
A

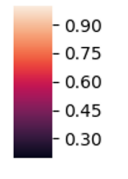

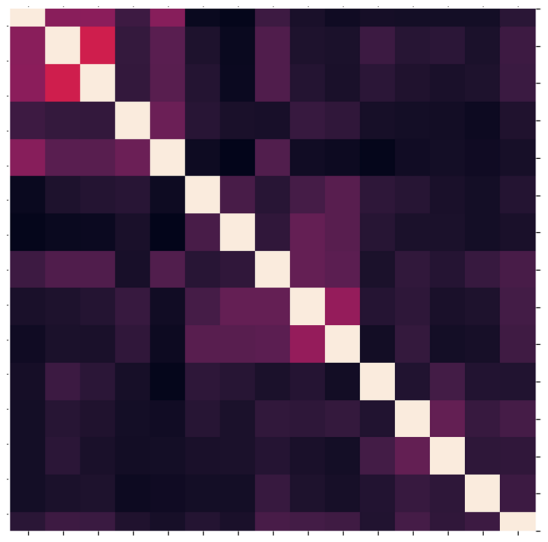

B

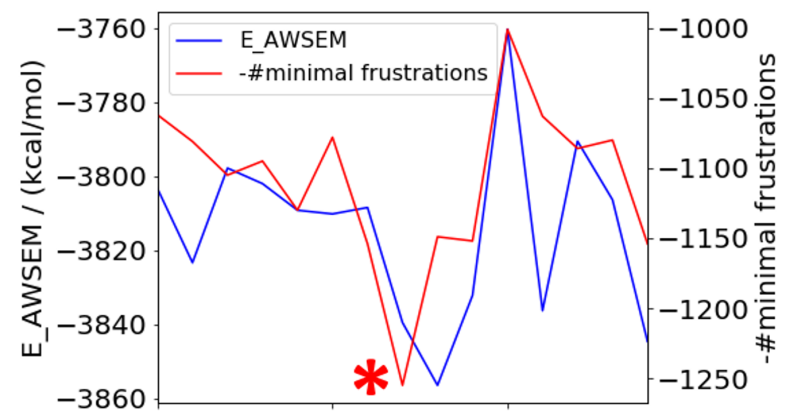

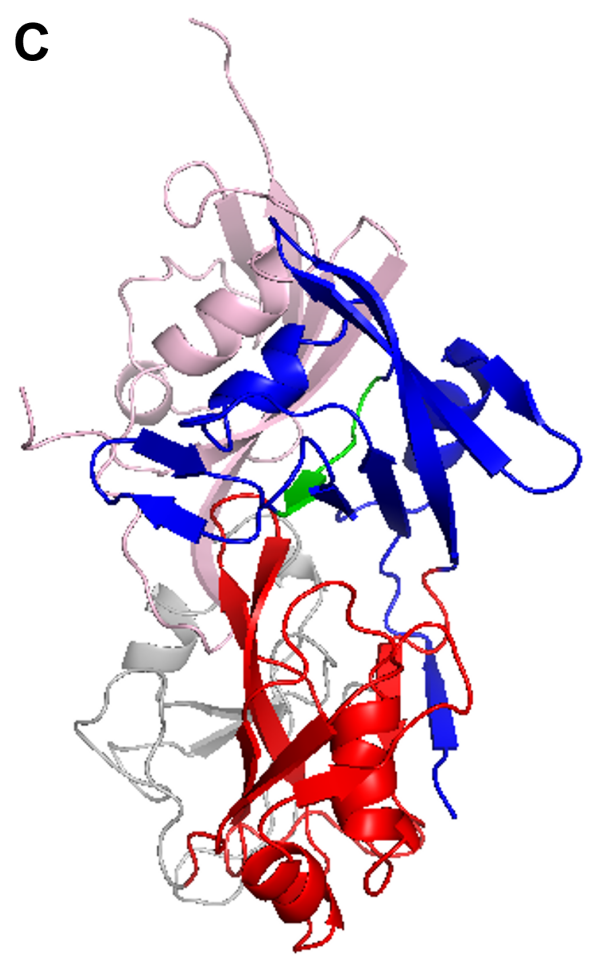

Figure 3: A. Clustering analysis for the predicted structures of the SUMO2/RBD complex using mutual Q (including inter-domain and inter-molecular residue pairs) as the structural similarity metric. B. Potential energy (blue line) and numbers of minimally frustrated contacts (red line) for the predicted SUMO2/RBD complex structures. The structure with the largest number of minimally frustrated contacts and having relatively low potential energy is selected as the representative structure (marked with a red star) and is shown in Figure C. C. The predicted structure of the SUMO2/RBD complex. The SUMO2 is colored in pink and the SIM in RRM1 is colored in green.

\section{The SUMO2/RBD interaction increases the RNA binding affinity of} the RBD

The specific binding of the RBD with SUMO2 makes the two RNA recognition motifs prefer the closed state, which is a favorable conformation for RNA binding. To further investigate the influence of this allosteric effect on the RNA-binding dynamics, we introduced a short piece of RNA containing 5 nucleotides to represent a target CPEB3 mRNA. We then used the AWSEM-3SPN2 force field along with an additional, sequence-specific protein-nucleic acid potential to simulate the combined protein/RNA system. The strength of the residue-nucleic acid potential for each base and residue pair was tuned by using the atomic contact numbers for each pair in the NMR structure of the RNA-bound RRMs as a reference. The NMR titration experiment shows that the RNA dissociation constant of RRMs is $15.8 \pm 6.6 \mu M,{ }^{23}$ which corresponds to an RNA-binding affinity of $6.4 \sim 6.9 \mathrm{kcal} / \mathrm{mol}$. In our simulations, 

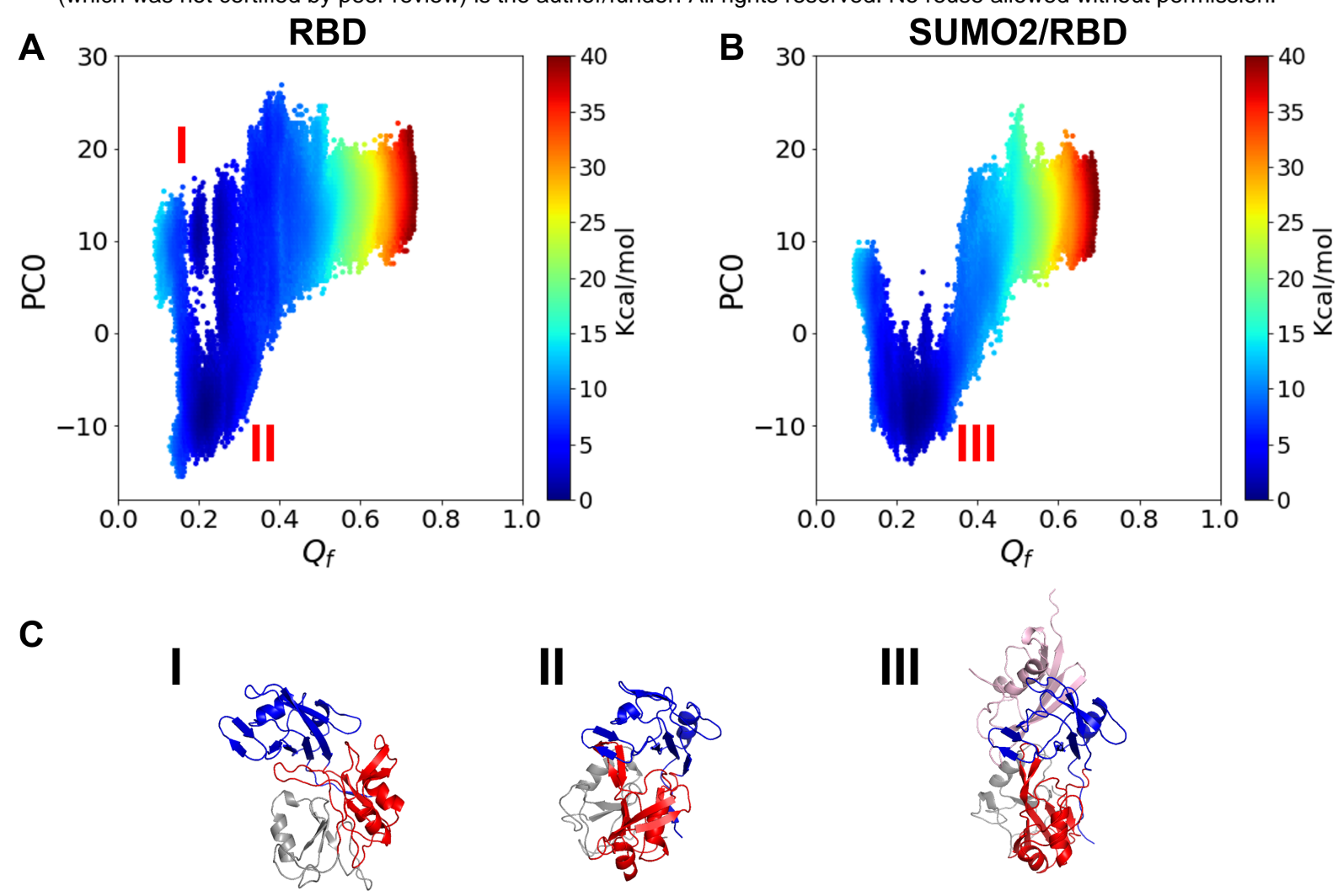

Figure 4: A. The free energy landscape for RBD plotted using $Q_{f}$ and $\mathrm{PC} 0$ as the two order parameters. $Q_{f}$ is the structural similarity metric to the NMR structure of free RRMs considering inter-domain residue pairs between RRM1 and RRM2 only. B. The free energy landscape for the SUMO2/RBD complex plotted using $Q_{f}$ and $\mathrm{PC} 0$ as the two order parameters. C. Representative structures for the three free energy basins in Figure A and B.

we calibrated the overall strength of the residue-nucleic acid potential to obtain a similar RNA-binding affinity, $8 \mathrm{kcal} / \mathrm{mol}$, for RRMs, as shown in Fig. 6A.

The 1D free energy profiles for the protein/RNA systems suggest that the RNA-binding affinity of the SUMO2/RBD complex is around $2 \mathrm{kcal} / \mathrm{mol}$ higher than the affinity of the RBD alone for RNA. (Fig. 5A). Upon projecting the free energy onto an additional order parameter, the principal component $\mathrm{PC} 0$, the $2 \mathrm{D}$ free energy profiles show a clear transition of dissociation pathway from one along path I to another along path II when SUMO2 becomes bound to the RBD (Fig. 5B). Along path I (Fig. 5C), the two RRMs open up during RNA dissociation, while along path II (Fig. 5D) they remain closed because of the SUMO2/RBD interaction. As sketched in Fig. 6, we propose that RNA dissociation from the RBD can be separated into two steps: first, RRM2 loses contact with the RNA and then rotates away from the binding pocket; following this, RRM1 dissociates from target RNA. For the SUMO2/RBD complex, however, it is difficult for RRM2 to dissociate from the RNA 
bioRxiv preprint doi: https://doi.org/10.1101/2021.11.24.469918; this version posted November 24,2021 . The copyright holder for this preprint (which was not certified by peer review) is the author/funder. All rights reserved. No reuse allowed without permission.
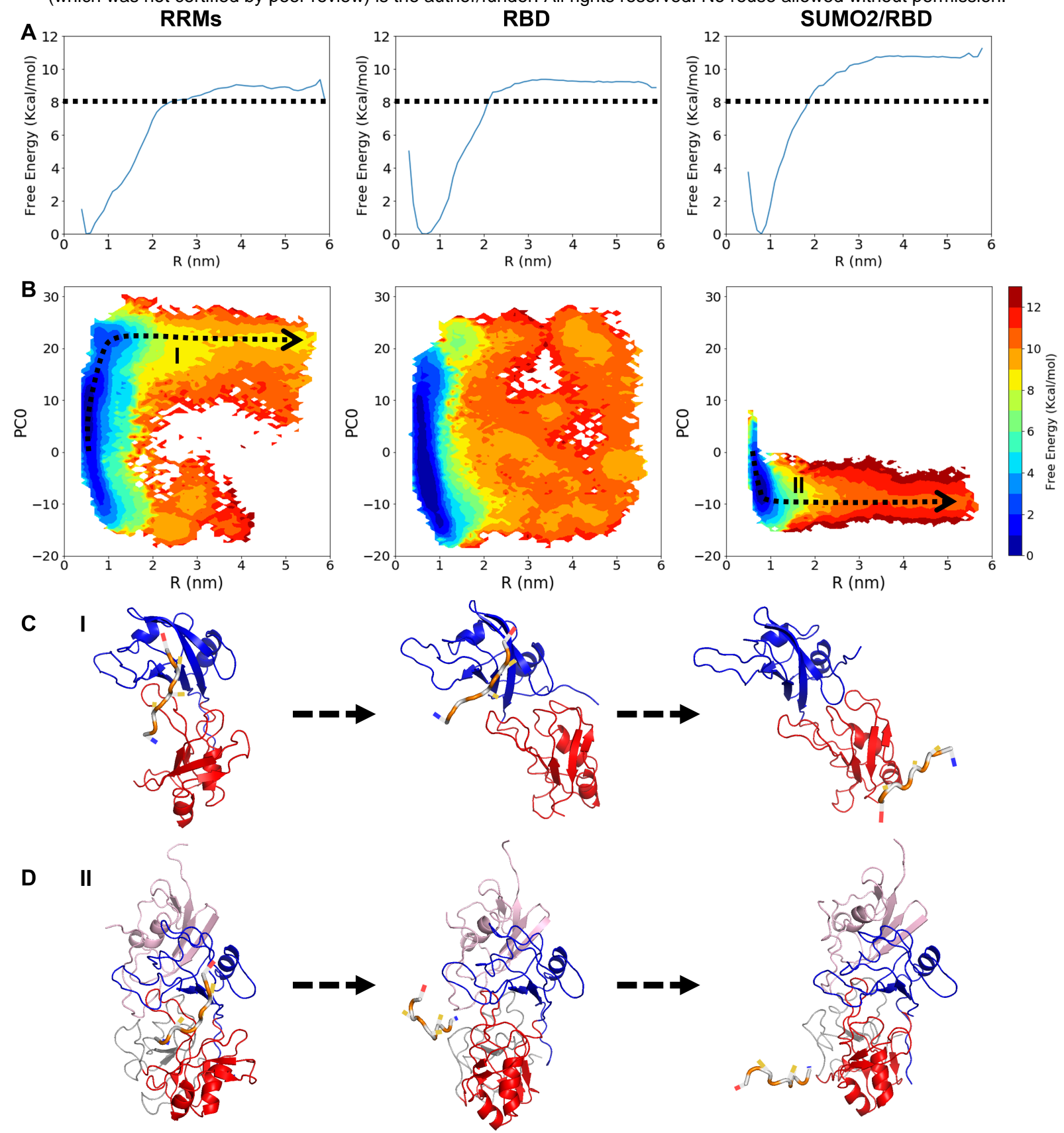

Figure 5: A. 1D free energy profiles for RNA dissociation from the RRMs (left), RBD (middle) and SUMO2/RBD complex (right), using R, the distance between RNA and the RNA binding pocket in RRMs, as the order parameter. The dashed black lines indicate a free energy difference of $8 \mathrm{kcal} / \mathrm{mol}$ from the RNA-bound state. B. 2D free energy landscapes for the RNA dissociation process from RRMs (left), RBD (middle) and SUMO2/RBD complex (right), using the principal component PC0 and R as two order parameters. C. Representative structures sampled from along the RNA dissociation pathway I from RRMs, as the dashed black arrow shown in Figure B. D. Representative structures sampled from along the RNA dissociation pathway II from SUMO2/RBD complex, as shown in Figure B. The main chain of the target RNA is colored in white and orange.

since RRM2 always stays in a ready-to-bind conformation when RRM1 is bound to RNA.

RNA dissociation from the SUMO2/RBD complex therefore requires RRM1 and RRM2 to dissociate simultaneously from the RNA, thereby raising the barrier to dissociation. The 

tandem RNA recognition motifs.

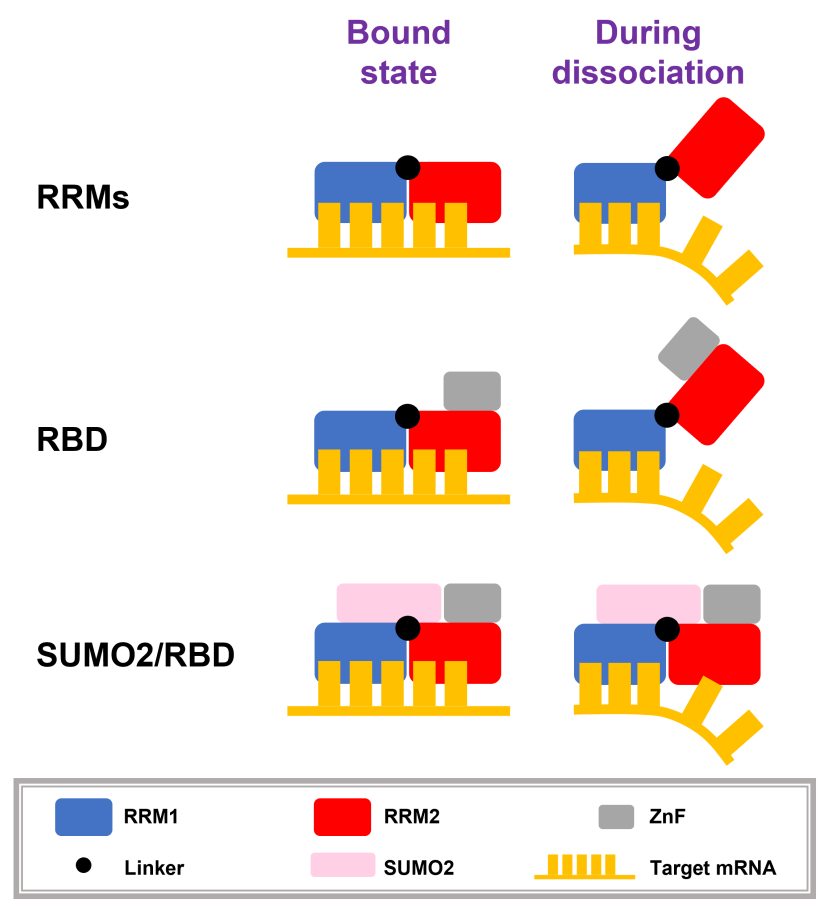

Figure 6: Schematic structural diagrams of the RNA dissociation processes from RRMs, RBD, and SUMO2/RBD. For the RRMs and the RBD, the RNA dissociation is a two-step process: RNA dissociation from RRM2 is followed by its dissociation from RRM1. For the SUMO2/RBD complex, RNA must dissociate from the two RRMs simultaneously. 

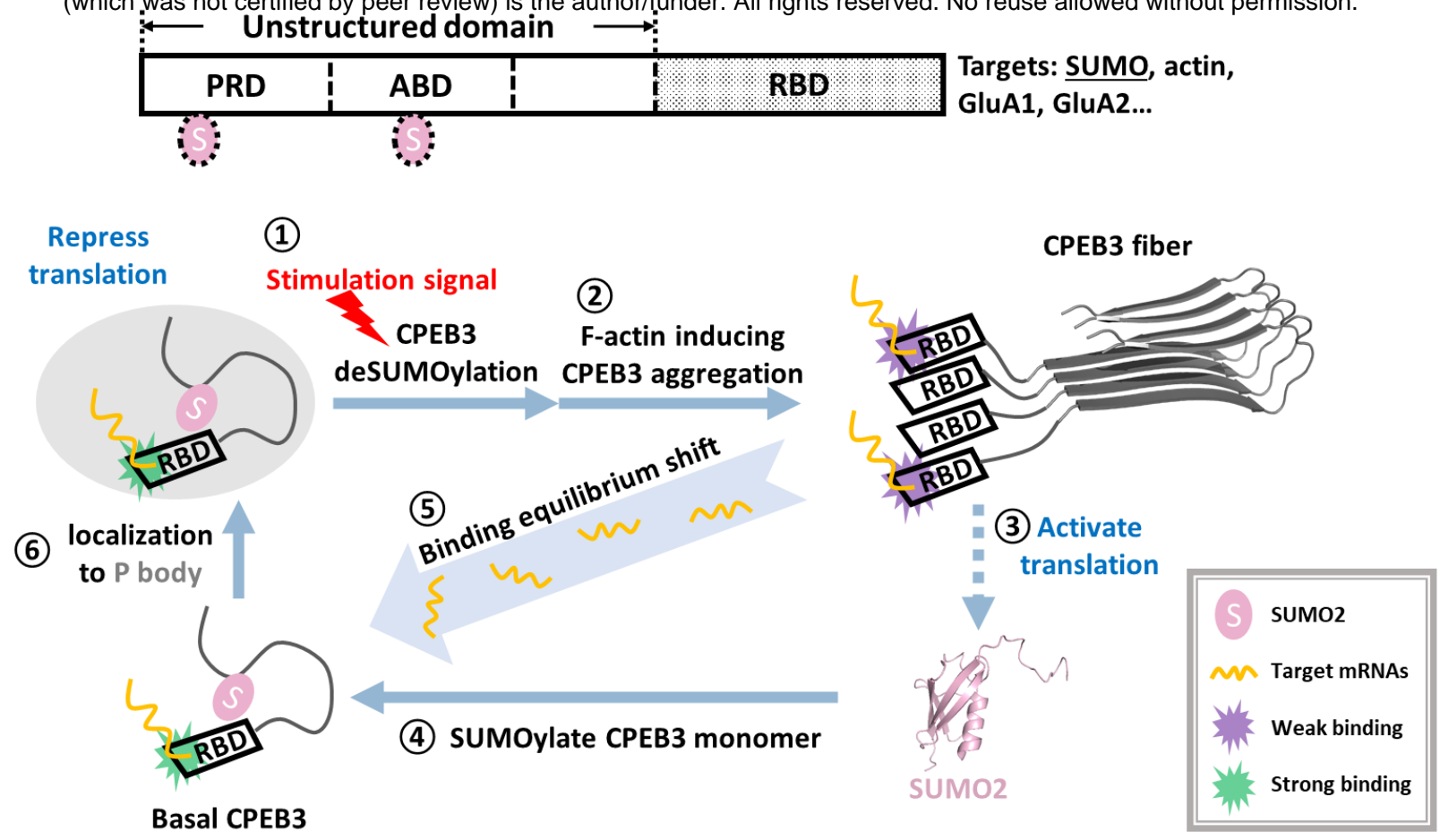

Figure 7: The two potential SUMOylated sites on CPEB3 are highlighted using two SUMO2 labels (pink) in the upper diagram. The complete negative feedback loop between CPEB3 and SUMO2 is illustrated in the lower panel: (1) An external signal stimulates the synapses and triggers the deSUMOylation of basal CPEB3. (2) CPEB3 exposes its prion-like domain $(\mathrm{PRD})$ and actin-binding domain $(\mathrm{ABD})$ and aggregates upon binding with actin filaments (F-actin). (3) CPEB3 fibers activate the translation of its target mRNAs, including SUMO2 mRNA. (4) Newly synthesized SUMO2 proteins are used for the SUMOylation of monomeric CPEB3. (5) An equilibrium shift of mRNAs from binding with CPEB3 fibers to binding with SUMOylated CPEB3 occurs due to the RNA-binding affinity difference. (6) SUMOylated CPEB3 binds with target mRNAs and recruits them into $\mathrm{P}$ bodies for translational repression. A legend is shown at the lower-right corner.

\section{Discussion}

\section{A negative feedback loop between CPEB3 and SUMO2 is completed}

\section{by an equilibrium shift of RNA binding}

The systems biology of SUMO2/CPEB3/mRNA suggests a negative feedback loop which addressed the potential problem of unregulated CPEB3 aggregation. SUMO2 mRNA, as a target of CPEB3, is activated by CPEB3 aggregates. While the translation products, SUMO2 proteins, can be attached to monomeric CPEB3 and prevent monomeric CPEB3 from further aggregation. CPEB3 aggregates which have already been formed still exist, however, after such a feedback loop. Therefore, the problem of unregulated translational enhancement by long-lasting CPEB3 aggregates still remains. 
regulating CPEB3's RNA-binding affinity, we see then that a more complete model of the

switch of CPEB3's translational control can be sketched (Fig. 7). Previous studies have proposed that the prion-like domain and the actin-binding domain of SUMOylated CPEB3 is buried based on bioinformatic searches for SUMOylation sites and SIMs in CPEB3. ${ }^{16}$ After stimulation, CPEB3 is deSUMOylated and its PRD and ABD are exposed. Actin filaments are then able to bind with the exposed CPEB3-ABD and facilitate CPEB3 aggregation. CPEB3 fibers promote the translation of SUMO2 proteins, which are used for SUMOylating monomeric CPEB3 in synapses. SUMOylated CPEB3, having a higher RNA-binding affinity than a CPEB3 fiber, recruits target mRNAs from the CPEB3 fibers and sequesters them into $\mathrm{P}$ bodies. In this way, the synapses return to a new basal state after a stimulation pulse. The extent of the equilibrium shift of mRNA binding upon SUMOylation determines the efficacy of the switch in CPEB3's function in translational control. Free energy profiles in Fig. 5A show that the difference of the RNA-binding affinity of the SUMO2/RBD complex and that of the RBD by itself is around $2 \mathrm{kcal} / \mathrm{mol}$. Therefore, in equilibrium, the ratio of repressed target mRNA binding with SUMOylated CPEB3 to active target mRNA binding with deSUMOylated CPEB3 fiber would be around 30 in basal state (see detailed discussion in SI text). Accordingly, the function of CPEB3 could be sufficiently switched back to translational repression in basal synapses.

More experiments will be required to test the predictions of our model. Measuring the binding affinity of SUMO2 to CPEB3-RBD and solving the structure of the SUMO2/CPEB3RBD complex would provide direct experimental tests of the calculations that we have performed. To assess whether the predicted SUMO2/RBD interaction is formed in full length CPEB3, the RNA-binding affinity of SUMOylated CPEB3 should be measured and compared with that of deSUMOylated CPEB3. Structural studies of monomeric CPEB3 and aggregated CPEB3 will be crucial to uncovering the functions of CPEB3 in long-term memory. 
This work was supported by the Center for Theoretical Biological Physics, sponsored by NSF grants CHE-1743392 and PHY-2019745. Additionally, we wish to recognize the D.R. Bullard Welch Chair at Rice University, Grant C-0016 (to Wolynes). X.G., N.P.S. and P.G.W. designed research; X.G. performed research; X.G., N.P.S., C.B., W.L. and P.G.W. contributed new analytic tools; X.G., N.P.S. and P.G.W. analyzed data; and X.G., N.P.S. and P.G.W. wrote the paper. The authors declare that they have no competing interests.

\section{References}

(1) Matus, A. Actin-based plasticity in dendritic spines. Science 2000, 290, 754-758.

(2) Bailey, C. H.; Kandel, E. R. Structural changes accompanying memory storage. Annual review of physiology 1993, 55, 397-426.

(3) Fioriti, L.; Myers, C.; Huang, Y.-Y.; Li, X.; Stephan, J. S.; Trifilieff, P.; Colnaghi, L.; Kosmidis, S.; Drisaldi, B.; Pavlopoulos, E., et al. The persistence of hippocampal-based memory requires protein synthesis mediated by the prion-like protein CPEB3. Neuron 2015, 86, 1433-1448.

(4) Si, K.; Giustetto, M.; Etkin, A.; Hsu, R.; Janisiewicz, A. M.; Miniaci, M. C.; Kim, J.-H.; Zhu, H.; Kandel, E. R. A neuronal isoform of CPEB regulates local protein synthesis and stabilizes synapse-specific long-term facilitation in aplysia. Cell 2003, 115, 893904.

(5) Stepien, B. K.; Oppitz, C.; Gerlach, D.; Dag, U.; Novatchkova, M.; Krüttner, S.; Stark, A.; Keleman, K. RNA-binding profiles of Drosophila CPEB proteins Orb and Orb2. Proceedings of the National Academy of Sciences 2016, 113, E7030-E7038.

(6) Mastushita-Sakai, T.; White-Grindley, E.; Samuelson, J.; Seidel, C.; Si, K. Drosophila Orb2 targets genes involved in neuronal growth, synapse formation, and protein turnover. Proceedings of the National Academy of Sciences 2010, 107, 11987-11992.

(7) Stephan, J. S.; Fioriti, L.; Lamba, N.; Colnaghi, L.; Karl, K.; Derkatch, I. L.; Kan- 
bioRxiv preprint doi: https://doi.org/10.1101/2021.11.24.469918; this version posted November 24, 2021. The copyright holder for this preprint toskeleton. Cell reports 2015, 11, 1772-1785.

(8) Raveendra, B. L.; Siemer, A. B.; Puthanveettil, S. V.; Hendrickson, W. A.; Kandel, E. R.; McDermott, A. E. Characterization of prion-like conformational changes of the neuronal isoform of Aplysia CPEB. Nature structural \& molecular biology 2013, 20, 495-501.

(9) Crick, F. Memory and molecular turnover. Nature 1984, 312, 101.

(10) Chazeau, A.; Giannone, G. Organization and dynamics of the actin cytoskeleton during dendritic spine morphological remodeling. Cellular and molecular life sciences $\mathbf{2 0 1 6}$ 73, 3053-3073.

(11) Khan, M. R.; Li, L.; Pérez-Sánchez, C.; Saraf, A.; Florens, L.; Slaughter, B. D.; Unruh, J. R.; Si, K. Amyloidogenic oligomerization transforms Drosophila Orb2 from a translation repressor to an activator. Cell 2015, 163, 1468-1483.

(12) Drisaldi, B.; Colnaghi, L.; Fioriti, L.; Rao, N.; Myers, C.; Snyder, A. M.; Metzger, D. J.; Tarasoff, J.; Konstantinov, E.; Fraser, P. E., et al. SUMOylation is an inhibitory constraint that regulates the prion-like aggregation and activity of CPEB3. Cell reports 2015, 11, 1694-1702.

(13) Ford, L.; Ling, E.; Kandel, E. R.; Fioriti, L. CPEB3 inhibits translation of mRNA targets by localizing them to P bodies. Proceedings of the National Academy of Sciences 2019, 116, 18078-18087.

(14) Hervas, R.; Rau, M. J.; Park, Y.; Zhang, W.; Murzin, A. G.; Fitzpatrick, J. A.; Scheres, S. H.; Si, K. Cryo-EM structure of a neuronal functional amyloid implicated in memory persistence in Drosophila. Science 2020, 367, 1230-1234.

(15) Gu, X.; Schafer, N. P.; Wolynes, P. G. Vectorial channeling as a mechanism for translational control by functional prions and condensates. Proceedings of the National Academy of Sciences 2021, 118.

(16) Gu, X.; Schafer, N. P.; Wang, Q.; Song, S. S.; Chen, M.; Waxham, M. N.; Wolynes, P. G. Exploring the F-actin/CPEB3 interaction and its possible role in the molecular mech- 
bioRxiv preprint doi: https://doi.org/10.1101/2021.11.24.469918; this version posted November 24, 2021. The copyright holder for this preprint $117,22128-22134$.

(17) Davtyan, A.; Schafer, N. P.; Zheng, W.; Clementi, C.; Wolynes, P. G.; Papoian, G. A. AWSEM-MD: protein structure prediction using coarse-grained physical potentials and bioinformatically based local structure biasing. The Journal of Physical Chemistry B 2012, 116, 8494-8503.

(18) Lu, W.; Bueno, C.; Schafer, N. P.; Moller, J.; Jin, S.; Chen, X.; Chen, M.; Gu, X.; Davtyan, A.; de Pablo, J. J., et al. OpenAWSEM with Open3SPN2: A fast, flexible, and accessible framework for large-scale coarse-grained biomolecular simulations. PLoS computational biology 2021, 17, e1008308.

(19) Onuchic, J. N.; Luthey-Schulten, Z.; Wolynes, P. G. Theory of protein folding: the energy landscape perspective. Annual review of physical chemistry 1997, 48, 545-600.

(20) Zheng, W.; Schafer, N. P.; Davtyan, A.; Papoian, G. A.; Wolynes, P. G. Predictive energy landscapes for protein-protein association. Proceedings of the National Academy of Sciences 2012, 109, 19244-19249.

(21) Hinckley, D. M.; Freeman, G. S.; Whitmer, J. K.; De Pablo, J. J. An experimentallyinformed coarse-grained 3-site-per-nucleotide model of DNA: Structure, thermodynamics, and dynamics of hybridization. The Journal of chemical physics 2013, 139, 10B604_1.

(22) Freeman, G. S.; Hinckley, D. M.; Lequieu, J. P.; Whitmer, J. K.; De Pablo, J. J. Coarse-grained modeling of DNA curvature. The Journal of chemical physics 2014, $141,10 \mathrm{~B} 615$ _.

(23) Afroz, T.; Skrisovska, L.; Belloc, E.; Guillén-Boixet, J.; Méndez, R.; Allain, F. H.-T. A fly trap mechanism provides sequence-specific RNA recognition by CPEB proteins. Genes \& Development 2014, 28, 1498-1514.

(24) Merkel, D. J.; Wells, S. B.; Hilburn, B. C.; Elazzouzi, F.; Pérez-Alvarado, G. C.; Lee, B. M. The C-terminal region of cytoplasmic polyadenylation element binding pro- 
bioRxiv preprint doi: https://doi.org/10.1101/2021.11.24.469918; this version posted November 24, 2021. The copyright holder for this preprint

biology 2013, 425, 2015-2026.

(25) Fiser, A.; Šali, A. Modeller: generation and refinement of homology-based protein structure models. Methods in enzymology 2003, 374, 461-491.

(26) Hake, L. E.; Mendez, R.; Richter, J. D. Specificity of RNA binding by CPEB: requirement for RNA recognition motifs and a novel zinc finger. Molecular and cellular biology 1998, 18, 685-693.

(27) Huang, Y.-S.; Kan, M.-C.; Lin, C.-L.; Richter, J. D. CPEB3 and CPEB4 in neurons: analysis of RNA-binding specificity and translational control of AMPA receptor GluR2 mRNA. The EMBO journal 2006, 25, 4865-4876.

(28) Kerscher, O. SUMO junction-what's your function? New insights through SUMOinteracting motifs. EMBO reports 2007, 8, 550-555.

(29) Danielsen, J. R.; Povlsen, L. K.; Villumsen, B. H.; Streicher, W.; Nilsson, J.; Wikström, M.; Bekker-Jensen, S.; Mailand, N. DNA damage-inducible SUMOylation of HERC2 promotes RNF8 binding via a novel SUMO-binding Zinc finger. Journal of Cell Biology 2012, 197, 179-187.

(30) Diehl, C.; Akke, M.; Bekker-Jensen, S.; Mailand, N.; Streicher, W.; Wikström, M. Structural analysis of a complex between small ubiquitin-like modifier 1 (SUMO1) and the $\mathrm{ZZ}$ domain of CREB-binding protein $(\mathrm{CBP} / \mathrm{p} 300)$ reveals a new interaction surface on SUMO. Journal of Biological Chemistry 2016, 291, 12658-12672.

(31) Parra, R. G.; Schafer, N. P.; Radusky, L. G.; Tsai, M.-Y.; Guzovsky, A. B.; Wolynes, P. G.; Ferreiro, D. U. Protein Frustratometer 2: a tool to localize energetic frustration in protein molecules, now with electrostatics. Nucleic acids research $\mathbf{2 0 1 6}$ 44, W356-W360. 